\title{
Positively increased visceral adiposity index in hyperuricemia free of metabolic syndrome
}

\author{
Dongfeng $\mathrm{Gu}^{{ }^{* *}}$, Yanan Ding ${ }^{2 \dagger}$, Yunfeng Zhao ${ }^{1}$, Shuzhai Miao ${ }^{1}$ and Qingshan Qu ${ }^{1}$
}

\begin{abstract}
Background: Visceral adiposity index (VAI) was closely associated with metabolic syndrome, however almost no research focused on VAl and hyperuricemia, therefore, this study was conducted to determine the relationship of VAI and hyperuricemia free of metabolic syndrome and estimate the power of VAl as predictor for hyperuricemia.

Methods: A cross-sectional research coming from a health check-up program was conducted. All participants were divided into four groups according to VAl quartiles. A multivariate logistic analysis was used to analyze the relationship between the quartiles and hyperuricemia. A receiver operating characteristic (ROC) curve analysis was used to evaluate the accuracy of predictions for hyperuricemia.

Results: VAI was independent risk factor of hyperuricemia. The ORs of which in the upper quartile were 3.077 (95\%Cl 1.78-5.293), $P=0.000$, in model 1, after adjusting for age, systolic blood pressure, diastolic blood pressure, heart rate, fast plasma glucose, serum creatinine, triglyceride, total cholesterol, high density lipoprotein cholesterol, and low density lipoprotein cholesterol; and 3.041 (95Cl 1.767-5.233), $P=0.000$, in model 2, after adjusting for the above plus physical activity, diet, smoking habits, alcohol consumption, hypertension and diabetes history. The area under the ROC curve (AUC) value of VAI was $0.618(95 \% \mathrm{Cl} 0.572-0.665), P=0.000$; it was higher than WC, which was 0.556 (95\%Cl 0.508-0.604), $P=0.024$, for hyperuricemia.
\end{abstract}

Conclusions: VAI was associated with hyperuricemia among individuals free of metabolic syndrome, and also a powerful indicator.

Keywords: Visceral adiposity index, Hyperuricemia, Metabolic syndrome, Obesity

\section{Background}

There are two kinds of central obesity: subcutaneous and visceral fat mass, and waist circumference (WC) alone does not help distinguishing them [1]. The Visceral Adiposity Index (VAI), which is based on WC, body mass index (BMI), triglycerides(TG), and high density lipoprotein cholesterol (HDL-C) and was recently introduced by Amato et al. [2], was used as a marker of visceral fat dysfunction, and been suggested as an indicator of Metabolic syndrome (MetS) [3, 4]. MetS, which was defined as a distinct entity which comprises the following components: central obesity,

\footnotetext{
* Correspondence: dongfenggu@hotmail.com

'Equal contributors

'Department of Nephrology and Transplantation Center, The People's Hospital of Zhengzhou, Affiliated with Southern Medical University, Zhengzhou 450003, People's Republic of China

Full list of author information is available at the end of the article
}

dyslipidemia, hyperglycemia and hypertension, increase a person's risk of cardiovascular disease [5, 6], and more and more studies revealed that hyperuricemia is a risk factor of MetS [7-9].

Serum uric acid is a clinically useful nutritional marker [10], and hyperuricemia has been a major health problem in the world; the prevalence of which was $9.9 \%$ in men (cutoff point $=7.0 \mathrm{mg} / \mathrm{dl}$ ) and $7.0 \%$ in women (cutoff point $=6.0 \mathrm{mg} / \mathrm{dl}$ ) according to a national cross-sectional survey in China [11], however the prevalence of serum urate levels $>6 \mathrm{mg} / \mathrm{dl}$ was $50.4 \%$ among men and $16.3 \%$ among women in US [12]. Asymptomatic hyperuricemia, may progress into gout which characterized as monosodium urate crystal deposition, and also was independently associated with prevalent hypertension [13]. Therefore, it has been a public health burden, especially in males.

(c) The Author(s). 2018 Open Access This article is distributed under the terms of the Creative Commons Attribution 4.0 International License (http://creativecommons.org/licenses/by/4.0/), which permits unrestricted use, distribution, and reproduction in any medium, provided you give appropriate credit to the original author(s) and the source, provide a link to the Creative Commons license, and indicate if changes were made. The Creative Commons Public Domain Dedication waiver (http://creativecommons.org/publicdomain/zero/1.0/) applies to the data made available in this article, unless otherwise stated. 
However, there was almost no research on the relationship of VAI and hyperuricemia, and MetS may be a confounder, therefore, we initiated to do this research about VAI and hyperuricemia free of MetS in Chinses males, in order to study the relationship of VAI and hyperuricemia free of metabolic syndrome and estimate the power of VAI as predictor for hyperuricemia.

\section{Methods}

\section{Participants}

A cross-sectional research from 3 Jan, 2016 to 3 Jan, 2017 was conducted. Sample size was determined by statistician, and which was more than 288 at a given confidence level of $5 \%$, confidence interval of $95 \%$, and central obesity epidemiology of $24.9 \%$ in China referred from Lancet [14]. The Ethics Committee of The People's Hospital of Zhengzhou approved the study. A total of 713 male adult participants were recruited from a selfpaid health check-up program at the Health Management Centre of The People's Hospital of Zhengzhou with individual informed consent, written or oral, according to the principles expressed in the Declaration of Helsinki. The information of their anthropometric measurements, biochemical data, medical history and medication usage were well documented; subjects with acute illness, malignancy, infection including of chronic hepatitis $\mathrm{B}$ and chronic hepatitis $\mathrm{C}$, current users of antithyroid, glucocorticoids, unclear cold medicine, and non-steroid anti-inflammatory drugs, were excluded, especially MetS. Finally, 633 non-MetS male participants were included in our analysis.

\section{Questionnaire}

Anthropometric measurements, medical history and medication usage were included in questionnaires as described in detail previously [15]. Participants were asked standing erect and had relaxed the abdominal muscles before WC measurement by a flexible inch tape. Measurement was taken at the end of normal expiration. Locate the top of the hip bone (iliac crest) and take the measurement just above this bony landmark, just where one finger can fit between the iliac crest and the lowest rib. The questionnaire consisted of questions regarding age, sex, a personal history of diabetes (yes vs. no), a personal history of hypertension (yes vs. no), a personal history of cardiovascular disease (yes vs. no), diet (balanced diet vs. unbalanced diet) according to Chinese Dietary Balance Index and Diet Quality Distance recommended by the Chinese Nutrition Society, Diet Quality Distance (range 0 to 84 ) was calculated based on DBI-07, 0-17 were looked as balanced diet, and 17-84 were looked as unbalanced diet [16], Physical activity (>60 $\mathrm{min} /$ day vs. 30-60 $\mathrm{min} /$ day vs. <30 $\mathrm{min} /$ day vs. no), > $60 \mathrm{~min} /$ day was considered as active physical activity, and $<30 \mathrm{~min} /$ day and no were considered as inactive physical activity based on our previous publication [15], smoking habits (yes[current] vs. no), alcohol intake (yes[current] vs. no); WC, height and systolic blood pressure (SBP) and diastolic blood pressure (DBP) were each measured manually [15]. Prior to BP measurements, participants were seated quietly for 5 to $10 \mathrm{~min}$ in a chair with arm supported at heart level and the rotator cuff positioned $3 \mathrm{~cm}$ above the antecubital fossa, BP was measured using Omron (SEM 1 Model) automatic BP monitor (Omron Healthcare Co., Ltd., IL, USA) with an appropriate cuff size $[17,18]$. Heart rate (HR) was also documented from the automatic BP monitor. Average BP and HR were then calculated from three measurements. BMIs were calculated using the following equation: BMI = weight $(\mathrm{kg}) /$ height $^{2}\left(\mathrm{~m}^{2}\right)$.

\section{Biochemical data}

Biochemical data acquiring Protocols was also described in detail in our previous publication [15]. Appointments were scheduled for blood collection. Fasting venous blood draws were performed at The People's Hospital of Zhengzhou. All blood samples were sent to the central laboratory of The People's Hospital of Zhengzhou. The blood samples were either disposed of within $3 \mathrm{~h}$ or stored at $4{ }^{\circ} \mathrm{C}$ for as long as 2 days. FPG (fasting plasma glucose, FPG) testing was performed via an electrochemical luminescence immunoassay. Serum total cholesterol (TC), HDLC, TG, and low-density lipoprotein cholesterol (LDLC) were each measured using an autoanalyzer (Toshiba, Japan). Serum creatinine (Scr) was measured using overnight fasting venous blood samples, via Jaffe's kinetic method. Uric acid was detected by uricase-based spectrophotometry.

\section{Evaluation criteria}

VAI, a sex-specific index based on WC, BMI, TG and HDLC, was calculated as follows [2]:

$$
\begin{aligned}
\text { Males : VAI }= & \left(\frac{\mathrm{WC}}{39.68+(1.88 \times B M I)}\right) \times\left(\frac{\mathrm{TG}}{1.03}\right) \\
& \times\left(\frac{1.31}{\mathrm{HDL}}\right) \\
\text { Females : VAI }= & \left(\frac{\mathrm{WC}}{36.58+(1.89 \times \mathrm{BMI})}\right) \times\left(\frac{\mathrm{TG}}{0.81}\right) \\
& \times\left(\frac{1.52}{\mathrm{HDL}}\right)
\end{aligned}
$$

MetS was defined as a composition of any 3 of 5 risk factors including of central obesity, raised triglycerides, lowered high-density lipoprotein cholesterol, raised fasting glucose and raised blood pressure [5, 6]. Hyperuricemia was defined as a serum uric acid (SUA) concentration 
exceeding the saturation point of $6.8 \mathrm{mg} / \mathrm{dL}(404 \mu \mathrm{mol} / \mathrm{L})$ at physiologic $\mathrm{pH}$ and body temperature, without symptoms from crystal deposition [19].

\section{Statistical analysis}

Acquired data were analyzed using SPSS 16.0 (SPSS Inc., Chicago, IL, USA). Continuous variables were represented as means \pm SDs, and categorical variables were represented as proportions of each group. The basic characteristics of the four VAI quartiles were examined in non-MetS males. Continuous variables were analyzed via one-way ANOVA, and categorical variables were analyzed via the Chi-square test or the Fisher's exact test.

Logistic regression models were used to determine whether VAI is associated with hyperuricemia free of MetS males. VAI was divided into four quartiles and considered a categorical variable. Model one was adjusted for age, VAI, SBP, DBP, heart rate, FPG, sCr, TC, TG, HDLC and LDLC; model two was adjusted for the above confounders, plus physical activity, diet, smoking habits, alcohol consumption, HP and DM. The lower quartile was used as a reference category. And logistic regression was also performed in analyzing the risk factor of VAI. $P$ values less than 0.05 were considered statistically significant.

A receiver operating characteristic ( $\mathrm{ROC}$ ) curve analysis was used to evaluate the accuracy of predictions for hyperuricemia free of MetS males. The accuracy was showed as the area under the ROC curve (AUC) with 95\% confidence interval (CI). To determine the appropriate cut-off point for each adiposity index, the score with the highest Youden's index (sensitivity+specificity-1, Youden's Index) was considered as the optimal cut-off one.

\section{Results}

\section{The basic characteristics of the male and female participants}

As shown in Table 1, there were significant differences in inactive physical activity, diabetes mellitus, WC, BMI, DBP, HR, SUC, serum glucose, TG, HDLC, TC, and LDLC in non-MetS males, as the upper VAI quartile participants exhibited higher DBP, serum glucose, TG, TC, and LDLC, prevalence of inactive physical activity, and lower HDLC, compared with the lower VAI subjects in non-MetS males.

\section{The relationship between VAI and hyperuricemia free of MetS males}

As shown in Table 2, the VAI was significantly associated with hyperuricemia free of MetS males, after adjusting for age, SBP, DBP, HR, FPG, sCr, TG, TC, HDLC, and LDLC; the ORs for hyperuricemia in the upper quartile of the VAI were 3.077 (95\%CI 1.78-5.293), $P=0.000$, in nonMetS males. Following further adjustments for the above confounders, physical activity, diet, smoking habits, alcohol consumption, HP and DM, the ORs for hyperuricemia in the upper quartile of the VAI were 3.041 (95CI 1. 767-5.233), $P=0.000$, in non-MetS males.

Table 3 summarized the ability of three indices to predict hyperuricemia free of MetS males. The AUC value of VAI was 0.618 (95\%CI $0.572-0.665), P=0.000$, and the cut-off values of which was 1.15 for hyperuricemia. The AUC value of $\mathrm{WC}$ was 0.556 (95\%CI $0.508-0.604), P=0$. 024, and the cut-off values of which was 79.5 for hyperuricemia. BMI has no significant meaning, $P=0.810$.

Figure 1 compared the accuracy of VAI, WC and BMI for predicting hyperuricemia free of MetS males. Both VAI and WC were positively correlated with hyperuricemia, and VAI had the highest AUC.

\section{Discussion}

The study revealed that there was a significantly relationship between VAI and hyperuricemia free of MetS males. Risk of hyperuricemia was higher in the upper quartile of VAI compared to the lower quartile of VAI, the OR was 3. 077 (1.789-5.293), $P=0.000$. Our pilot study also performed a multivariate logistic regression using VAI values as continuous variables, which revealed that VAI was also independent risk factor of hyperuricemia free of MetS males, the OR was $2.811(2.128-3.714), P=0.000$. VAI had the highest AUC compared with WC and BMI in hyperuricemia free of MetS males, which was not revealed in the literature to the best of our knowledge.

Some anthropometric indices of obesity had been studied to explore the relationship of which with hyperuricemia. Body shape index (BSI), body roundness index (BRI), BMI, WC and waist-to -height ratio (WHtR) were significantly associated with hyperuricemia in both males and females, and BRI rather than ABSI showed a superior predictive ability for identifying hyperuricemia than BMI in female and similar capabilities as those of WC and WHtR in the female, but not in the male gender [20]. BMI increase and hypertriglyceridemia may potentiate serum uric acid effect on gout development [21]. Another Chinese research found that WC was strongly correlated with hyperuricemia in the Asian Mongolian area [22]. One research revealed that metabolically healthy obese, which means obese individuals with a favorable metabolic profile, had a high risk for hyperuricemia among Chinese general population [23, 24]. All of these studies were not excluded of MetS, and it was still controversial which one of anthropometric indices was superior to others. However, our research reveals that VAI was superior to $\mathrm{WC}$ in predicting of hyperuricemia free of MetS.

VAI not only dramatically increased with SUA, but also significantly increased with DBP, and FPG, as shown in Table 1 . This has been already verified in literatures. VAI was significantly increased in 
Table 1 Baseline characteristics of Non-metabolic syndrome health check-up population

\begin{tabular}{|c|c|c|c|c|c|}
\hline & \multicolumn{4}{|l|}{ VAI (male) } & \multirow[t]{2}{*}{$P$} \\
\hline & $\begin{array}{l}\text { 1st Quartile } \\
(n=162)\end{array}$ & $\begin{array}{l}\text { 2nd Quartile } \\
(n=162)\end{array}$ & $\begin{array}{l}\text { 3rd Quartile } \\
(n=151)\end{array}$ & $\begin{array}{l}\text { 4th Quartile } \\
(n=158)\end{array}$ & \\
\hline Age (years) & $53.3 \pm 16.6$ & $54.4 \pm 15.0$ & $51.8 \pm 13.8$ & $52.7 \pm 13.5$ & 0.458 \\
\hline Inactive Physical Activity (\%) & $93(57.4 \%)$ & $96(59.3 \%)$ & $102(67.5 \%)$ & $117(74.1 \%)$ & $P<0.05$ \\
\hline Unbalanced Diet (\%) & $39(24.1 \%)$ & $38(23.5 \%)$ & $39(25.8 \%)$ & $46(29.1 \%)$ & 0.655 \\
\hline Current Smoking (\%) & $47(29.0 \%)$ & $55(34.0 \%)$ & $38(25.2 \%)$ & 63(39.9\%) & 0.331 \\
\hline Current Alcohol (\%) & $25(15.4 \%)$ & $21(13.0 \%)$ & $22(14.6 \%)$ & $26(16.5 \%)$ & 0.842 \\
\hline Hypertension (\%) & $33(20.4 \%)$ & $37(22.8 \%)$ & $37(24.5 \%)$ & $50(31.6 \%)$ & 0.111 \\
\hline Diabetes Mellitus (\%) & $74(4.3 \%)$ & $6(3.7 \%)$ & $17(11.3 \%)$ & 16(10.1\%) & $P<0.05$ \\
\hline Waist Circumference (cm) & $79.7 \pm 8.6$ & $85.8 \pm 8.7$ & $90.0 \pm 8.1$ & $91.3 \pm 7.9$ & $P<0.001$ \\
\hline Body Mass Index (kg/m²) & $21.9 \pm 2.8$ & $23.4 \pm 3.0$ & $24.6 \pm 3.2$ & $25.1 \pm 2.7$ & $P<0.001$ \\
\hline Systolic Blood Pressure (mmHg) & $128.0 \pm 20.4$ & $129.8 \pm 18.3$ & $129.7 \pm 17.0$ & $132.3 \pm 18.2$ & 0.227 \\
\hline Diastolic Blood Pressure (mmHg) & $76.5 \pm 11.1$ & $78.0 \pm 10.2$ & $80.6 \pm 9.9$ & $82.2 \pm 9.6$ & $P<0.001$ \\
\hline Heart rate (/min) & $73.7 \pm 11.3$ & $72.6 \pm 9.8$ & $74.5 \pm 9.6$ & $76.8 \pm 11.0$ & $P<0.05$ \\
\hline Serum Creatinine $(\mu \mathrm{mol} / \mathrm{L})$ & $85.5 \pm 14.2$ & $85.4 \pm 15.3$ & $88.7 \pm 15.1$ & $89.0 \pm 16.4$ & 0.051 \\
\hline Serum uric acid (mmol/L) & $370.9 \pm 83.0$ & $385.4 \pm 82.9$ & $402.0 \pm 83.9$ & $431.0 \pm 89.3$ & $P<0.001$ \\
\hline Serum glucose (mmol/L) & $4.8 \pm 1.1$ & $5.0 \pm 1.2$ & $5.2 \pm 1.2$ & $5.4 \pm 1.6$ & $P<0.05$ \\
\hline Triacylglycerols(mmol/L) & $0.8 \pm 0.2$ & $1.1 \pm 0.2$ & $1.8 \pm 0.4$ & $3.4 \pm 1.6$ & $P<0.001$ \\
\hline Total Cholesterol (mmol/L) & $5.1 \pm 1.0$ & $5.3 \pm 0.9$ & $5.5 \pm 1.0$ & $5.5 \pm 0.9$ & $P<0.001$ \\
\hline High-Density Lipoprotein Cholesterol (mmol/L) & $1.7 \pm 0.3$ & $1.4 \pm 0.2$ & $1.3 \pm 0.2$ & $1.3 \pm 0.3$ & $P<0.001$ \\
\hline Low-Density Lipoprotein Cholesterol (mmol/L) & $3.0 \pm 0.8$ & $3.4 \pm 0.9$ & $3.3 \pm 0.9$ & $3.9 \pm 0.9$ & $P<0.01$ \\
\hline
\end{tabular}

Means \pm SDs represented the continuous variables, and proportions represented the categorical variables

1st quartile of VAl: 0-0.78; 2nd quartile of VAl: 0.79-1.18; 3rd quartile of VAl: 1.19-1.90; 4th quartile of VAl: $\geq 1.90$

Continuous variables were analyzed via One-way ANOVA, categorical variables were analyzed via the Chi-square test or Fisher's exact test, and $P$ value less than 0.05 was considered statistical significant

prehypertension and hypertension in our previous report [15]. Some studies also found that VAI was associated with diabetes [25-27]. However, the underlying mechanism is puzzling. SUA was characterized as both pro-oxidant and antioxidant properties, depending on the context, and it can impair endothelial function, result to atherosclerotic risk [28-30]. New founding was that Visceral fat, not subcutaneous depot, exhibited greater expression of proinflammatory, oxidative stress-related, hypoxia-induced, and proangiogenic genes; increased activated macrophage

Table 2 The relationship between visceral adiposity index and hyperuricemia free of metabolic syndrome males

\begin{tabular}{llllll}
\hline VAl & Model one $^{\text {a }}$ & & & Model two $^{{ }^{b}}$ \\
\cline { 2 - 3 } & OR $(95 \% \mathrm{Cl})$ & $P$ & & OR $(95 \% \mathrm{Cl})$ & $P$ \\
\hline 1st Quartile & Reference & & Reference & \\
2nd Quartile & $1.513(0.896-2.556)$ & 0.121 & & $1.514(0.896-2.557)$ & 0.121 \\
3rd Quartile & $2.101(1.246-3.542)$ & 0.005 & & $2.102(1.247-3.543)$ & 0.005 \\
4th Quartile & $3.077(1.789-5.293)$ & 0.000 & & $3.041(1.767-5.233)$ & 0.000
\end{tabular}

a Adjusted for age, SBP, DBP, heart rate, FPG, sCr, triglyceride, total cholesterol, high density lipoprotein cholesterol, and low density lipoprotein cholesterol; ${ }^{{ }^{\prime}}$ Adjusted for the above plus physical activity, diet, smoking habits, alcohol consumption, $\mathrm{HP}$ and DM populations; and had a higher capacity for cytokine production ex vivo; thus providing clinical evidence that visceral microenvironment play key important role in atherosclerotic vascular disease [31]. Excess visceral adiposity induces alterations in mitochondrial function and energy metabolism in tumor [32], and correlated with the expression of genes related to inflammation and oxidative stress in peripheral blood cells [31]. Visceral fat adiposity was associated with sub-clinical inflammation and increased oxidative stress [33]. Therefore, the mechanism of VAI intermediated hyperuricemia was remained to be revealed.

Inactive physical activity has positively with VAI in our study. As is known dyslipidemia was the basic of VAI increase. One Mexican population study revealed that sweetened beverages intake increased the risk of hyperuricemia and obesity [34]. Maybe keep fit and diet control was helpful for visceral obesity, specially in Chinese males, since Chinese individuals had the most deleterious abdominal visceral fat distribution and accumulation other than Europeans at a given BMI or WC [35]. A prospective study should be conducted to identify their causal relationship. However, it's time to control visceral obesity. 
Table 3 The power of VAI, WC and BMI in hyperuricemia free of metabolic syndrome males

\begin{tabular}{llllllll}
\hline & AUC & $(95 \% \mathrm{Cl})$ & $P$ value & Cut off & Sensitivity & 1-Specificity & Yonden's index \\
\hline VAl & 0.618 & $0.572-0.665$ & 0.000 & 1.15 & 0.628 & 0.428 & 0.200 \\
WC & 0.556 & $0.508-0.604$ & 0.024 & 79.5 & 0.781 & 0.655 & 0.126 \\
BMI & 0.494 & $0.445-0.543$ & 0.810 & 19.05 & 0.939 & 0.901 & 0.038 \\
\hline
\end{tabular}

Interleukin-6 (IL-6), tumor necrosis factor-alpha (TNF- $\alpha$ ), and high sensitive C-reactive protein (hs-CRP) were also measured in order to determine the relationship of indices of inflammation with VAI and hyperuricemia. Our research also revealed that IL-6, TNF- $\alpha$, and hs-CRP levels are significantly increased with hyperuricemia (IL-6: $r=0.308, P<0.05$; TNF- $\alpha: r=0.365, P<0$. 05; hs-CRP: $r=0.420, P<0.01$ ), and also have positive correlation with VAI (IL-6: $r=0.348, P<0.05$; TNF- $\alpha$ : $r=0.405, P<0.05$; hs-CRP: $r=0.496, P<0.01$ ). It' well known that adipocytes can synthesize TNF- $\alpha$ and IL-6, the synthesis of $\mathrm{C}$-reactive protein (CRP) in hepatocytes is IL-6 dependent, and CRP is also associated with adipose tissue [36]. High-fat and/or calorie-rich diet may also enhance production of these proinflammatory cytokines [37]. VAI has been associated with an increase in IL-6, TNF- $\alpha$, hs-CRP in obese patients [38]. TNF- $\alpha$ has effects on TG through adipose tissue and liver TG metabolic pathways by increasing the level of proinflammatory cytokines such as IL-6 [38, 39]. It well documented that serum uric acid was also positively associated with IL-6, TNF- $\alpha$, and hs-CRP [40-42]. Therefore, adiposity may be an inflammatory condition. However, the underlying mechanism is still to be revealed in the future.

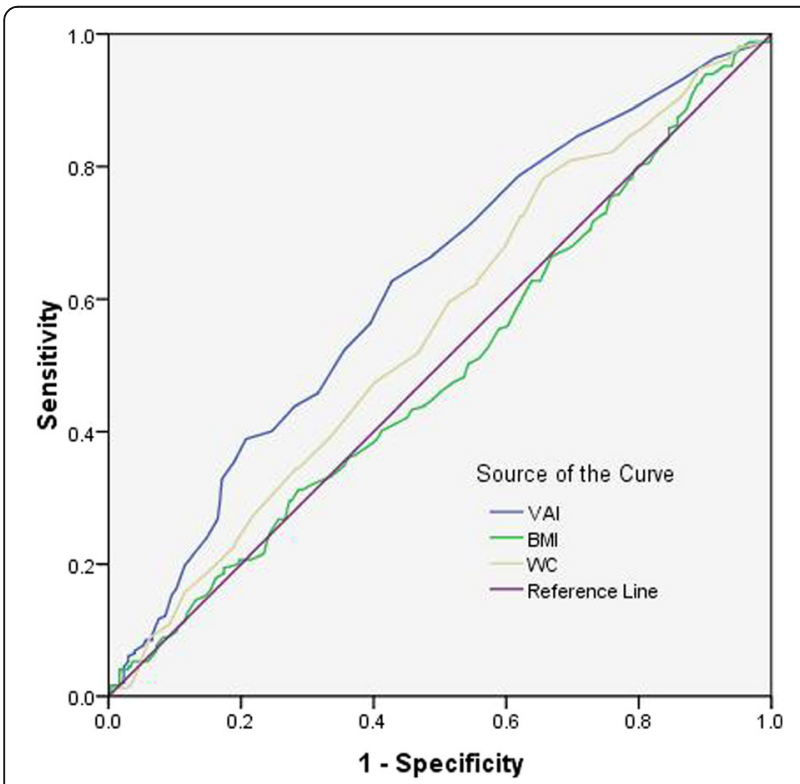

Fig. 1 The discriminatory power of VAI, WC and BMI in asymptomatic hyperuricemia free of metabolic syndrome males

\section{Limitations}

The VAI was established in Caucasian populations, its suitability for other populations needs to be further confirmed. It remains to develop a well-designed epidemiological study to determine the underling mechanism.

\section{Conclusions}

VAI was independently associated with hyperuricemia among individuals free of MetS and also a powerful indicator.

\section{Abbreviations}

AUC: Area under the ROC; BMI: Body mass index; BRI: Body roundness index; BSI: Body shape index; Cl: Confidence interval; CRP: C-reactive protein; DBP: Diastolic blood pressure; DM: Diabetic mellitus; FPG: Fasting plasma glucose; HDL-C: High density lipoprotein cholesterol; HP: Hypertension; HR: Heart rate; hs-CRP: High sensitive C-reactive protein; IL-6: Interleukin-6; LDL-C: Low-density lipoprotein cholesterol; MetS: Metabolic syndrome; OR: Odds ratio; ROC: Receiver operating characteristic; SBP: Systolic blood pressure; Scr: Serum creatinine; SUA: Serum uric acid; TC: Total cholesterol; TG: Triglycerides; TNF-a: Tumor necrosis factor-alpha; VAl: Visceral adiposity index; VAT: Visceral adiposity; WC: Waist circumference; WHtR: Waist to height ratio

\section{Acknowledgements}

We thank all of the individuals who participated in the study, and are particularly grateful to the doctors and nursed involved in this study.

\section{Funding}

This study was supported by The People's Hospital of Zhengzhou. The funders had no role in study's design, data collection, decision to publish, or preparation of the manuscript.

\section{Availability of data and materials}

The datasets obtained and/or analyzed in the current study are available on reasonable requests from the corresponding author.

\section{Authors' contributions}

QQ conceived the original idea for the study and undertook its design with the assistance of each of the authors. YD and DG performed research and wrote the first draft of the manuscript; $Y Z$ and SM performed research and participated in the statistical analysis. Each of the authors read and approved the final manuscript.

\section{Ethics approval and consent to participate}

The study was approved by the ethics committee of the People's Hospital of Zhengzhou, and written informed consent was obtained from all participants.

\section{Consent for publication}

All authors provide consent for publication of this paper.

\section{Competing interests}

The authors declare that they have no competing interests.

\section{Publisher's Note}

Springer Nature remains neutral with regard to jurisdictional claims in published maps and institutional affiliations. 


\section{Author details}

'Department of Nephrology and Transplantation Center, The People's Hospital of Zhengzhou, Affiliated with Southern Medical University, Zhengzhou 450003, People's Republic of China. ${ }^{2}$ Division of Cardiovascular Medicine, The People's Hospital of Zhengzhou, Affiliated with Southern Medical University, Zhengzhou 450003, People's Republic of China.

\section{Received: 12 December 2017 Accepted: 26 April 2018}

\section{Published online: 07 May 2018}

\section{References}

1. Pouliot MC, Despres JP, Lemieux S, Moorjani S, Bouchard C, Tremblay A, Nadeau A, Lupien PJ. Waist circumference and abdominal sagittal diameter: best simple anthropometric indexes of abdominal visceral adipose tissue accumulation and related cardiovascular risk in men and women. Am J Cardiol. 1994;73(7):460-8.

2. Amato MC, Giordano C, Galia M, Criscimanna A, Vitabile S, Midiri M, Galluzzo A. Visceral adiposity index: a reliable indicator of visceral fat function associated with cardiometabolic risk. Diabetes Care. 2010;33(4):920-2.

3. Qing L, Wei R, Chan L, Xiaoya Z, Xin X. Sensitivity of various body indices and visceral adiposity index in predicting metabolic syndrome among Chinese patients with adult growth hormone deficiency. J Endocrinol Investig. 2017;40(6):653-61.

4. Motamed N, Khonsari MR, Rabiee B, Ajdarkosh H, Hemasi GR, Sohrabi MR, Maadi M, Zamani F. Discriminatory ability of visceral adiposity index (VAl) in diagnosis of metabolic syndrome: a population based study. Exp Clin Endocrinol. 2017;125(3):202-7.

5. Expert Panel on Detection, Evaluation, and Treatment of High Blood Cholesterol in Adults. Executive summary of the third report of the National Cholesterol Education Program (NCEP) expert panel on detection, evaluation, and treatment of high blood cholesterol in adults (adult treatment panel III). JAMA. 2001;285(19):2486-97.

6. Alberti KG, Eckel RH, Grundy SM, Zimmet PZ, Cleeman Jl, Donato KA, Fruchart JC, James WP, Loria CM, Smith SC Jr. Harmonizing the metabolic syndrome: a joint interim statement of the international diabetes federation task force on epidemiology and prevention; National Heart, Lung, and Blood Institute; American Heart Association; world heart federation; international atherosclerosis society; and International Association for the Study of obesity. Circulation. 2009;120(16):1640-5.

7. Zhang Q, Lou S, Meng Z, Ren X. Gender and age impacts on the correlations between hyperuricemia and metabolic syndrome in Chinese. Clin Rheumatol. 2011;30(6):777-87.

8. Nagahama K, Inoue T, Kohagura K, Ishihara A, Kinjo K, Ohya Y. Hyperuricemia predicts future metabolic syndrome: a 4-year follow-up study of a large screened cohort in Okinawa, Japan. Hypertens Res. 2014;37(3):232-8.

9. Wei CY, Sun CC, Wei JC, Tai HC, Sun CA, Chung CF, Chou YC, Lin PL, Yang T. Association between hyperuricemia and metabolic syndrome: an epidemiological study of a labor force population in Taiwan. Biomed Res Int. 2015;2015:369179.

10. Beberashvili I, Sinuani I, Azar A, Shapiro G, Feldman L, Stav K, Sandbank J, Averbukh Z. Serum uric acid as a clinically useful nutritional marker and predictor of outcome in maintenance hemodialysis patients. Nutrition. 2015; 31(1):138-47.

11. Liu H, Zhang XM, Wang YL, Liu BC. Prevalence of hyperuricemia among Chinese adults: a national cross-sectional survey using multistage, stratified sampling. J Nephrol. 2014;27(6):653-8.

12. Zhu Y, Pandya BJ, Choi HK. Prevalence of gout and hyperuricemia in the US general population: the National Health and nutrition examination survey 2007-2008. Arthritis Rheum. 2011:63(10):3136-41.

13. Krishnan E. Interaction of inflammation, hyperuricemia, and the prevalence of hypertension among adults free of metabolic syndrome: NHANES 20092010. J Am Heart Assoc. 2014;3(2):e000157.

14. Tian $Y$, Jiang $C$, Wang $M$, Cai $R$, Zhang $Y$, He Z, Wang H, Wu D, Wang F, Liu $X$, et al. BMI, leisure-time physical activity, and physical fitness in adults in China: results from a series of national surveys, 2000-14. Lancet Diab Endocrinol. 2016;4(6):487-97.

15. Ding Y, Gu D, Zhang Y, Han W, Liu H, Qu Q. Significantly increased visceral adiposity index in prehypertension. PLoS One. 2015;10(4):e0123414.

16. Stookey JD, Wang Y, Ge K, Lin H, Popkin BM. Measuring diet quality in China: the INFH-UNC-CH diet quality index. Eur J Clin Nutr. 2000;54(11): $811-21$.
17. National High Blood Pressure Education Program Working Group on High Blood Pressure in Children and Adolescents. The fourth report on the diagnosis, evaluation, and treatment of high blood pressure in children and adolescents. Pediatrics. 2004;114(2 Suppl 4th Report):555-76.

18. Pickering TG, Hall JE, Appel L, Falkner BE, Graves J, Hill MN, Jones DW, Kurtz T, Sheps SG, Roccella EJ. Recommendations for blood pressure measurement in humans and experimental animals: part 1: blood pressure measurement in humans: a statement for professionals from the Subcommittee of Professional and Public Education of the American Heart Association Council on high blood pressure research. Hypertension. 2005; 45(1):142-61.

19. Hamburger M, Baraf HS, Adamson TC 3rd, Basile J, Bass L, Cole B, Doghramji PP, Guadagnoli GA, Hamburger F, Harford R, et al. 2011 Recommendations for the diagnosis and management of gout and hyperuricemia. Postgrad Med. 2011;123(6 Suppl 1):3-36.

20. Zhang N, Chang Y, Guo X, Chen Y, Ye N, Sun Y. A body shape index and body roundness index: two new body indices for detecting association between obesity and hyperuricemia in rural area of China. Eur J Intern Med. 2016;29:32-6.

21. Chen JH, Pan WH, Hsu CC, Yeh WT, Chuang SY, Chen PY, Chen HC, Chang $C T$, Huang WL. Impact of obesity and hypertriglyceridemia on gout development with or without hyperuricemia: a prospective study. Arthritis Care Res. 2013;65(1):133-40.

22. You L, Liu A, Wuyun G, Wu H, Wang P. Prevalence of hyperuricemia and the relationship between serum uric acid and metabolic syndrome in the Asian Mongolian area. J Atheroscler Thromb. 2014;21 (4):355-65.

23. Chen $Y$, Zhang N, Sun G, Guo X, Yu S, Yang H, Zheng L, Sun Y. Metabolically healthy obesity also has risk for hyperuricemia among Chinese general population: a cross-sectional study. Obes Res Clin Pract. 2016;10(Suppl 1): S84-95.

24. Liu L, Lou S, Xu K, Meng Z, Zhang Q, Song K. Relationship between lifestyle choices and hyperuricemia in Chinese men and women. Clin Rheumatol. 2013;32(2):233-9.

25. Chen C, Xu Y, Guo ZR, Yang J, Wu M, Hu XS. The application of visceral adiposity index in identifying type 2 diabetes risks based on a prospective cohort in China. Lipids Health Dis. 2014:13:108.

26. Du T, Sun X, Huo R, Yu X. Visceral adiposity index, hypertriglyceridemic waist and risk of diabetes: the China health and nutrition survey 2009. Int J Obes. 2014:38(6):840-7.

27. Wang Y, He S, He J, Wang S, Liu K, Chen X. Predictive value of visceral adiposity index for type 2 diabetes mellitus: a 15-year prospective cohort study. Herz. 2014:40(suppl 3):277-81.

28. Krishnan E. Inflammation, oxidative stress and lipids: the risk triad for atherosclerosis in gout. Rheumatology (Oxford). 2010;49(7):1229-38.

29. Nieto FJ, Iribarren C, Gross MD, Comstock GW, Cutler RG. Uric acid and serum antioxidant capacity: a reaction to atherosclerosis? Atherosclerosis. 2000:148(1):131-9.

30. Ames BN, Cathcart R, Schwiers E, Hochstein P. Uric acid provides an antioxidant defense in humans against oxidant- and radical-caused aging and cancer: a hypothesis. Proc Natl Acad Sci U S A. 1981;78(11):6858-62.

31. Yamaoka M, Maeda N, Nakamura S, Kashine S, Nakagawa Y, Hiuge-Shimizu A, Okita K, Imagawa A, Matsuzawa Y, Matsubara K, et al. A pilot investigation of visceral fat adiposity and gene expression profile in peripheral blood cells. PLoS One. 2012;7(10):e47377.

32. Lynam-Lennon N, Connaughton R, Carr E, Mongan AM, O'Farrell NJ, Porter RK, Brennan L, Pidgeon GP, Lysaght J, Reynolds JV, et al. Excess visceral adiposity induces alterations in mitochondrial function and energy metabolism in esophageal adenocarcinoma. BMC Cancer. 2014;14:907.

33. Gariballa S, Kosanovic M, Yasin J, El Essa A. Oxidative damage and inflammation in obese diabetic Emirati subjects. Nutrients. 2014;6(11): 4872-80.

34. Lopez-Molina R, Parra-Cabrera S, Lopez-Ridaura R, Gonzalez-Villalpando ME, Ferrannini E, Gonzalez-Villalpando C. Sweetened beverages intake, hyperuricemia and metabolic syndrome: the Mexico City diabetes study. Salud Publica de Mexico. 2013;55(6):557-63.

35. Nazare JA, Smith JD, Borel AL, Haffner SM, Balkau B, Ross R, Massien C, Almeras N, Despres JP. Ethnic influences on the relations between abdominal subcutaneous and visceral adiposity, liver fat, and cardiometabolic risk profile: the international study of prediction of intraabdominal adiposity and its relationship with Cardiometabolic risk/intraabdominal adiposity. Am J Clin Nutr. 2012;96(4):714-26. 
36. Bahceci M, Gokalp D, Bahceci S, Tuzcu A, Atmaca S, Arikan S. The correlation between adiposity and adiponectin, tumor necrosis factor alpha, interleukin6 and high sensitivity C-reactive protein levels. Is adipocyte size associated with inflammation in adults? J Endocrinol Investig. 2007;30(3):210-4.

37. Das UN. Is there a role for bioactive lipids in the pathobiology of diabetes mellitus? Front Endocrinol (Lausanne). 2017:8:182.

38. Stepien M, Stepien A, Wlazel RN, Paradowski M, Banach M, Rysz J. Obesity indices and inflammatory markers in obese non-diabetic normo- and hypertensive patients: a comparative pilot study. Lipids Health Dis. 2014;13:29.

39. Popa C, Netea MG, van Riel PL, van der Meer JW, Stalenhoef AF. The role of TNF-alpha in chronic inflammatory conditions, intermediary metabolism, and cardiovascular risk. J Lipid Res. 2007;48(4):751-62.

40. Wasilewska A, Tenderenda E, Taranta-Janusz K, Tobolczyk J, Stypulkowska J. Markers of systemic inflammation in children with hyperuricemia. Acta Paediatr. 2012:101(5):497-500

41. Lyngdoh T, Marques-Vidal P, Paccaud F, Preisig M, Waeber G, Bochud M, Vollenweider P. Elevated serum uric acid is associated with high circulating inflammatory cytokines in the population-based Colaus study. PLoS One. 2011;6(5):e19901.

42. Di Y, Wang J, Chen Y, Sun N, Wu L, Dai X, Shen M, Ying Q, Fu S. Elevated Interleukin 1 beta and interleukin 6 levels in the serum of children with hyperuricemia. J Clin Rheumatol. 2018;24(2):65-9.

Ready to submit your research? Choose BMC and benefit from:

- fast, convenient online submission

- thorough peer review by experienced researchers in your field

- rapid publication on acceptance

- support for research data, including large and complex data types

- gold Open Access which fosters wider collaboration and increased citations

- maximum visibility for your research: over $100 \mathrm{M}$ website views per year

At BMC, research is always in progress.

Learn more biomedcentral.com/submissions 